Address for Correspondence: Dr. Xiao-chun Ma, Department of Critical Care Medicine, the First Affiliated Hospital of China Medical University, Shenyang 110001, Liaoning Province,

China.

Email: xcma2972@sina.com

\begin{tabular}{|c|}
\hline Access this article 0 \\
\hline $\begin{array}{l}\text { Website: } \\
\text { www.intern-med.com }\end{array}$ \\
\hline $\begin{array}{l}\text { DOI: } \\
\text { 10.1515/jtim-2017-0034 }\end{array}$ \\
\hline Quick Response Code: \\
\hline 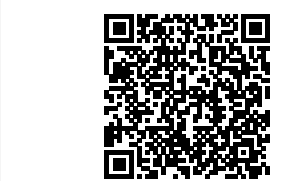 \\
\hline
\end{tabular}

\title{
Clinical value of ultrasonography in diagnosis of pulmonary embolism in critically ill patients
}

\author{
Ran Zhu ${ }^{1,2}$, Xiao-Chun Ma ${ }^{1,2}$ \\ 'Department of Critical Care Medicine, the First Affiliated Hospital of China Medical University, \\ Shenyang 110001, Liaoning Province, China; \\ ${ }^{2}$ Critical Care Ultrasound Study Group, Beijing, China
}

\section{ABSTRACT}

Pulmonary embolism (PE) is a clinical emergency that will increase the mortality if complicated with unstable hemodynamics. Because of its nonspecific clinical symptoms, it's a great challenge to make a PE diagnosis. The golden standard to diagnose PE is computed tomography of pulmonary artery (CTPA), but a diagnosis of PE also composed of evaluation of PE risk factors, possibilities, and risk stratification. Ultrasonography may detect right ventricle strain related to hemodynamic change, intravascular thrombosis, thrombosis in right heart or pulmonary arteries, pulmonary infarction, and local pleural effusion. Combination of ultrasound and traditional PE possibility evaluation score may further improve the pretest probability of CTPA. A comprehensive ultrasonography may sometimes rule out PE and may disclose other causes for the clinical situations. A heart-lung-vessel-integrated multiorgan ultrasonography can help with the diagnosis of PE and so should be a necessary weapon for the physicians.

Key words: pulmonary embolism; ultrasonography; critical care medicine

\section{INTRODUCTION}

Pulmonary embolism (PE) can cause a series of clinical manifestation. It could occur in patients from different departments. With its acute onset and quick progress, $\mathrm{PE}$ is one of the most lethal diseases. However, the diagnosis of $\mathrm{PE}$ is still a great challenge for the clinicians. All suspected patients need to be ruled in or ruled out $\mathrm{PE}$ in order to accept definite treatments, no matter they are critically ill patients complicated with a newly onset PE, or becoming critically ill because of respiratory and circulatory failure caused by PE. The 2014 ESC Guidelines on the Diagnosis and Management of Acute Pulmonary Embolism already included cardiac ultrasonography in the diagnosis and treatment procedures of high-risk patients ${ }^{[1]}$; however, PE can be diagnosed or ruled out easily if we adopt critical care ultrasonography strategy to perform a goal-oriented, multiorgan, point of care (POC) ultrasonography on probable PE patients rather than limited to cardiac ultrasonography. PE can also be treated under the guidance of ultrasonography. This paper is intended to describe the ultrasound images of PE and the ultrasound diagnosis strategy of PE.

\section{GOAL-ORIENTED, MULTIORGAN, POC ULTRASONOGRAPHY}

\section{Indirect ultrasound signs of PE in heart and great blood vessel}

The pathophysiology of circulatory failure in patients with high-risk PE is the increased pulmonary vascular resistance due to pulmonary vascular obstruction and vasospasm, causing right ventricular pressure overload and dysfunctioned, which may further reduce left ventricular preload and output; pressure overload in right ventricle may increase the ventricular wall tension, causing abnormal coronary blood flow, and further deteriorate the right ventricular function; right ventricle dysfunction may restrict the filling of left 
ventricle through interventricular septum and pericardium, and then further decrease the left ventricular output. In clinical practice, fluid resuscitation shall be initiated according to different types of shock, otherwise the volumetric load of right ventricle will further deteriorate right ventricle and left ventricle. ${ }^{[1]}$ On the basis of these pathophysiological changes, for patients with probable PE complicated with unstable hemodynamics, the ultrasonic examination shall be aimed at finding signs of increased right ventricular afterload, for example, right ventricular enlargement, "D" sign and abnormal motion in interventricular septum, and restricted left ventricular filling; meanwhile, the inferior vena cava should be fixed and expanded because of restricted venous return to the right heart. ${ }^{[2]}$

Other than the static ultrasound signs mentioned above, the right ventricle may show signs of abnormal systolic function when the right ventricular pressure overload is not relived within a short time, such as reduced tricuspid annular motion and reduced ejection fraction of the right ventricle. A special ultrasound signs of right ventricular abnormal systolic function, named "McConnell's sign," was proposed in 1996, characterized by reduced right ventricular free wall motion but reserved apical motion. After its proposal, the McConnell's sign was believed to be the specific sign of acute PE for a time, which shall be able to distinguish acute right ventricular strain caused by $\mathrm{PE}$ from chronic right ventricular strain caused by pulmonary hypertension (PAH). Some retrospective or prospective studies even confirmed a 100\% specificity of PE diagnosis using McConnell's sign. ${ }^{[3]}$ However, a later study compared the imaging of patients with PE and patients with right ventricular infarction only to find that there was no statistical significance in the incidence of McConnell's sign between those two groups. ${ }^{[4]}$ Another retrospective study showed that with all the patients showing McConnell's sign, only half of them were proved to have PE at last. ${ }^{[5]}$ In addition to the right ventricular shape and motion abnormality, because of the changes in the pressure between chambers, patients with PE can also show signs of tricuspid regurgitation under Doppler ultrasound examination; tricuspid regurgitation can be used to estimate pulmonary artery systolic pressure and pulmonary vascular resistance. ${ }^{[2]}$

Before looking for those acute and prominent ultrasound signs, any chronic heart abnormality must be first ruled out. For instance, chronic cor pulmonale, often complicated with chronic obstructive pulmonary disease (COPD) and chronic pulmonary thromboembolism (CPTE) caused by gradual increase in right ventricle loading, may show similar ultrasound signs, such as right ventricular enlargement, interventricular septum shift to the left, restricted left ventricular filling, right ventricular motion abnormalities, and inferior vena cava expanded and fixed; However, the chronic change may lead to the right ventricular wall thickening and right atrial enlargement. ${ }^{[2]}$ We should learn to distinguish $\mathrm{PE}$ ultrasound signs from chronic right heart diseases, otherwise the ultrasonic diagnosis of PE may be misleading.

\section{Direct ultrasound signs of PE in heart and great blood vessel}

A few patients with PE may show signs of embolus under ultrasound examination, such as straddling thrombus at pulmonary artery bifurication, ${ }^{[6]}$ or floating embolus in the right heart system (e.g., within inferior vena cava, right atrium, right ventricle, and pulmonary artery). Although these signs are helpful for the diagnosis of PE, they are not very common. And sometimes it is hard to display the pulmonary artery clearly because of the limitations of ultrasound. Signs of thrombus in the right heart system or pulmonary artery do not correlate with hemodynamic instability. And this kind of direct but less common sign is not the main target when diagnosing probable $\mathrm{PE}$ in patients using ultrasound.

\section{Indirect ultrasound signs of PE in lungs and pleura}

Ultrasound examination of both lungs and pleura may show pulmonary consolidation caused by peripheral PE, which typically shows as a wedge-shape or round-shape hypo-echo subpleural image, sometimes accompanied with local pleural effusion. A study of transthoracic ultrasonography in patients with acute probable PE made a semiquantitative statistical analysis on the ultrasonic images of lungs and pleura. The results showed that when the patients had high WELLS scores (i.e., high PE probability), the specificity of the diagnosis is extremely high if the transthoracic ultrasonography found typical signs of PE; when the patients had low WELLS scores (i.e., low PE probability), the negative predictive value is extremely high if the transthoracic ultrasonography showed a negative result or only a small amount of typical PE signs. ${ }^{[7]}$ Another study performed contrast-enhanced ultrasonography on the infarcted lung tissues of patients with confirmed PE. The results turned out that about $40 \%$ of patients showed no enhancement in lesion area, while $40 \%$ of patients showed heterogeneous enhancement in lesion area. These results indicate that contrast-enhanced ultrasonography on lungs may improve the diagnostic specificity of PE. ${ }^{[8]}$ If the diagnosis of $\mathrm{PE}$ is made solely under the guidance of lung and pleural ultrasonography, there are a few things to be noted: susceptible lobe or segment of pulmonary infarction; effects of ribs on transthoracic ultrasonography implementation; without consideration of central PE; spontaneous thrombolysis, 
and revascularization, which may change transthoracic ultrasonography images.

\section{Direct ultrasound signs of PE in proximal deep vein}

The formation of proximal deep venous thrombosis (DVT) is closely related to the development of PE. Therefore, a DVT history, WELLS score, or proximal DVT examination can improve the diagnostic efficiency of PE. A recent study showed that for patients with probable DVT, the diagnostic sensitivity and specificity can be up to $77.8 \%$ and $91.4 \%$, respectively, if a 3 -point venous compression ultrasound examination (common femoral vein, proximal great saphenous vein, and popliteal vein) was performed by trained emergency physicians. ${ }^{[9]}$ Obviously, ultrasound is very helpful for DVT assessment. However, any venous compression may increase the risk of thrombus falling off, ${ }^{[10]}$ especially for the newly formed free-floating thrombus. We must keep this in mind in the process of examination.

\section{Goal-oriented multiorgan POC}

Ultrasound signs of PE include direct and indirect signs. Direct signs, such as thrombus in the right heart and pulmonary artery, are of extremely high specificity but could not be the major targets because of its low detection rate. Indirect signs with high detection rate are the main screening targets for patients presenting with high risk $\mathrm{PE}$, such as signs of changes in right ventricle and interventricular septum. However, we should distinguish these signs from signs of chronic pulmonary heart disease. Wedge-shape or round-shape hypo-echo subpleural image and signs of local pleural effusion only exists in patients with peripheral PE. In order to find these signs, we need to search each intercostal space carefully. As mentioned in China Expert Consensus on Critical Ultrasound, a heart-lung-vessel-integrated ultrasonography can increase the diagnosis accuracy of PE. ${ }^{[11]}$ Therefore, during the diagnosis of PE, a bedside goal-oriented "heart-lungvessel" multiorgan ultrasound examination can increase the detection rate of PE-related signs and increase the diagnostic sensitivity up to $90 \%$. On the other hand, if the multiorgan ultrasound examination shows no signs of abnormality, the negative predictive value to rule out PE can be up to $95 \%$.

\section{PE POSSIBILITY EVALUATION COMBINED WITH ULTRASONOGRAPHY}

In order to increase the diagnostic specificity and accuracy, we recommend to take assistant measures to access the prior probability of PE before implementing computed tomography pulmonary angiography (CTPA), such as screening for risk factors and clinical indicators. WELLS Scoring System and Geneva Scoring System are the two common possibility evaluation systems for PE, including two aspects: assessment of signs and symptoms related to DVT; possibilities of any other diagnosis besides PE. Previous research has shown that WELLS score combined with lung and venous ultrasound increase the sensitivity and specificity of PE probability score by $12 \%$ and $22 \%$, respectively; and the need for CTPA in the diagnosis process is significantly reduced if further combined with D-Dimer. ${ }^{[12]}$ In addition to an early and rapid diagnosis of PE, a quick differentiation of types of shock is also crucial for ICU physicians. For patients with probable highrisk PE (with shock or unstable hemodynamics), PEinduced obstructive shock can be ruled out immediately if ultrasound shows no signs of right ventricular strain abnormality, interventricular septum flattening, or right ventricular systolic abnormality.

\section{DIAGNOSTIC PROTOCOL OF PE COMBINED WITH ULTRASONOGRAPHY}

The adoption of a diagnostic protocol can often minimize missed diagnosis and narrow down the etiology, such as the diagnosis protocol for shock or sudden cardiac arrest. There are two types of PE diagnostic protocol combined with ultrasound, one is in search for the probability of $\mathrm{PE}$ and the other is in search for the etiology of dyspnea.

PE Diagnostic Protocol in search for PE Probability: For patients with probable PE and unstable hemodynamics (complicated with shock or hypotension, i.e., high mortality risk), as mentioned in the 2014 ESC Guidelines on the Diagnosis and Management of Acute Pulmonary Embolism, if CTPA is not available, cardiac ultrasound assessment shall be implemented to seek for signs of increase in right ventricular strain. A negative ultrasound in these patients could rule out PE but there must be other reasons for the unstable hemodynamics which we need to keep looking for. If with a positive ultrasound finding, CTPA examination shall be arranged or PE-specific revascularization shall be considered. The 2014 ESC Guidelines did not mention ultrasound use in the diagnostic protocol for patients without unstable hemodynamics (not complicated with shock or hypotension, i.e., low and medium mortality risk). However, ultrasonography is still helpful in this circumstance. Vascular and pleural ultrasound could increase the negative predictive value of PE probability scoring. And cardiac ultrasound examination may show direct signs of thrombus in the right heart system 
or signs of right ventricular involvement but within its compensation range. ${ }^{[1]}$

PE Diagnostic Protocol in search for the etiology of dyspnea, "BLUE" Protocol, ${ }^{[13]}$ as a well-established fast lung ultrasound protocol, allows a quick diagnosis for respiratory failure (pneumonia, pulmonary edema, PE, pneumothorax, COPD/bronchial asthma). Both its diagnostic sensitivity and specificity for respiratory failure can almost be as high as $100 \%$. The diagnostic strategy of BLUE protocol is as follows: first, rule out the ventilation and gas exchange dysfunction in pulmonary alveoli; then rule out the vascular factors; and then COPD or bronchial asthma shall be taken into consideration. Therefore, if ultrasound show signs of increased extravascular lung water, reduced lung inflation, or less movement in either lungs, the first reasons to be considered for dyspnea (or hypoxia) may be exudative lesion (lung edema, pneumonia) or pneumothorax. If pleural sliding is symmetrical in both lungs and with good lung inflation, the above reasons could be excluded and we need to look for other reasons outside pulmonary alveoli. For example, if ultrasound shows signs of intravascular thrombosis, PE can be confirmed. If ultrasound shows negative results of lungs and vessels, COPD or bronchial asthma shall be taken into consideration. However, the BLUE Protocol does not make the most of goal-oriented multiorgan POC ultrasonography. Respiratory failure is often complicated with circulatory failure in critically ill patients. Therefore, a differential diagnosis should be based on a multiangle evaluation. Critical Consultation Ultrasonic Examination (CCUE) Protocol ${ }^{[14]}$ can make differential diagnosis under ultrasonic guidance looking into many different aspects, such as chronic cardiac disease, acute cardiac disease, volume status, pulmonary disorders, and intravascular thrombosis in $5 \mathrm{~min}$.

\section{REAL-TIME ULTRASOUND EVALUATION DURING PE TREATMENT}

Reperfusion therapy is critical for patients with unstable PE. A real-time ultrasound guidance may indicate the necessity and efficacy of the therapy. Ultrasonic signs as follows maybe an indication of effective treatment: previous right ventricle dilation alleviated; "D" shape of interventricular septum diminished; inferior vena cava no longer expanded and fixed; thrombus in the right heart system, or deep vein dissolved gradually. The indirect dynamic assessment of pulmonary artery pressure with ultrasonography may benefit the long-term follow-up evaluation; and it is also very helpful to find out if the patients are complicated with chronic thromboembolic pulmonary hypertension (CTEPH).

\section{ENDOBRONCHIAL ULTRASONOGRAPHY AND TRANSESOPHAGEAL ECHOCARDIOGRAPHY}

Endobronchial ultrasonography (EBUS) is mainly used for minimal invasive biopsy of bronchoscopical mediastinal lymph nodes and masses. Several cases reported accidental detection of thrombus in the main pulmonary artery or its main branches of patients with non-suspect PE during EBUS examination.

One case even reported that when implementing EBUS on patient with probable PE seeking for signs of central $\mathrm{PE}$, signs of sub-segmental $\mathrm{PE}$ in posterior basal segment of bilateral lower lungs were accidentally detected; the diagnosis of PE was confirmed by CTPA. These case reports indicate that EBUS may have potential value for the diagnosis of PE under abnormal circumstances. ${ }^{[15-16]}$

Although transesophageal echocardiography (TEE) is not the preferred ultrasonic examination for PE, it's been used more and more as an intraoperative monitoring method in some highrisk surgeries. Some case reports showed accidental detection of thrombus in the right heart system during intraoperative monitoring by TEE. ${ }^{[17]}$ Under these circumstances, a complete assessment for PE can also be conducted by TEE and a final diagnosis can be made based on that.

In 2015 and 2016, there were 2 cases (not reported) of non-cardiac surgery patients in our hospital who were accidentally detected with thrombus in the right heart and pulmonary artery when using intraoperative monitoring by TEE but with stable hemodynamics.

\section{CONCLUSION}

PE is a clinical emergency in need of rapid identification. But without specific clinical symptoms and in need of CTPA as a golden diagnostic tool, PE diagnosis becomes a great challenge for ICU or respiratory physicians. Bedside critical care ultrasound is a real-time POC examination with many advantages, such as rapidity and noninvasion. When combined with traditional WELLS scoring system, critical care ultrasound may help to improve the prior probability of CTPA or even decrease the demand for CTPA in unstable patients. A heart-lung-vessel-integrated multiorgan POC ultrasonography can confirm or rule out the diagnosis of probable PE in patients and helps to identify other diseases with similar clinical manifestation, such as cardiac tamponade. In conclusion, the goal-oriented multiorgan POC ultrasonic protocol should become an essential skill for clinicians during PE diagnosis. 


\section{Conflict of Interest}

None declared.

\section{REFERENCES}

1. Konstantinides SV, Torbicki A, Agnelli G, Danchin N, Fitzmaurice D, Galiè N, et al; Task Force for the Diagnosis and Management of Acute Pulmonary Embolism of the European Society of Cardiology (ESC). 2014 ESC guidelines on the diagnosis and management of acute pulmonary embolism. Eur Heart J 2014; 35: 3033-69.

2. Borloz MP, Frohna WJ, Phillips CA, Antonis MS. Emergency department focused bedside echocardiography in massive pulmonary embolism. J Emerg Med 2011; 41: 658-60.

3. Dresden S, Mitchell P, Rahimi L, Leo M, Rubin-Smith J, Bibi S, et al. Right ventricular dilatation on bedside echocardiography performed by emergency physicians aids in the diagnosis of pulmonary embolism. Ann Emerg Med 2014; 63: 16-24.

4. Casazza F, Bongarzoni A, Capozi A, Agostoni O. Regional right ventricular dysfunction in acute pulmonary embolism and right ventricular infarction. Eur J Echocardiogr 2005; 6: 11-4.

5. Vaid U, Singer E, Marhefta GD, Kraft WK, Baram M. Poor positive predictive value of McConnell's sign on transthoracic echocardiography for the diagnosis of acute pulmonary embolism. Hosp Pract 1995; 41: $23-7$.

6. Secko M, Legome E, Rinnert S. Saddle embolism diagnosed by pointof-care transthoracic echocardiography before computed tomography angiogram of the chest. Am J Emerg Med 2016; 34: 2467.

7. Abootalebi A, Golshani K, Karami M, Masoumi B, Aliasgharlou M. Diagnostic validity of ultrasonography in evaluation of pulmonary thromboembolism. Adv Biomed Res 2016, 5: 4.

8. Bartelt S, Trenker C, Görg C, Neesse A. Contrast-enhanced ultrasound of embolic consolidations in patients with pulmonary embolism: A pilot study. J Clin Ultrasound 2016; 44: 129-35.
9. Crowhurst TD, Dunn RJ. Sensitivity and specificity of three-point compression ultrasonography performed by emergency physicians for proximal lower extremity deep venous thrombosis. Emerg Med Australas 2013; 25: 588-96.

10. Mehdipoor G, Shabestari AA, Lip GY, Bikdeli B. Pulmonary Embolism as a Consequence of Ultrasonographic Examination of Extremities for Suspected Venous Thrombosis: A Systematic Review. Semin Thromb Hemost 2016; 42: 636-41.

11. Wang X, Liu D, Yu K, Guan X, Ma X, Yan J, et al.; China Critical Care Ultrasound Study Group (CCUSG); Critical Hemodynamic Therapy Collaborative Group (CHTC group). [Chinese Experts' Consensus on Critical Ultrasonography]. Zhonghua Nei Ke Za Zhi 2016; 55: 900-12.

12. Nazerian P, Volpicelli G, Gigli C, Becattini C, Papa GF, Grifoni S, et al. Diagnostic Performance of Wells Score Combined With Point-of-care Lung and Venous Ultrasound in Suspected Pulmonary Embolism. Acad Emerg Med 2017; 24: 270-80.

13. Lichtenstein DA, Mezière GA. Relevance of lung ultrasound in the diagnosis of acute respiratory failure: the BLUE protocol. Chest 2008; 134: 117-25.

14. Wang X, Zhao H, Liu D, Zhang H, Du W, Chai W, et al.; China Critical Ultrasound Study Group (CCUSG). The Effects of Peking Union Medical College Hospital Critical Ultrasonic management Scheme on the Etiological Diagnosis of Dyspnea and/or Hemodynamic Instability in ICU Patients. Zhonghua Nei Ke Za Zhi 2014; 53: 793-8.

15. Segraves JM, Daniels CE. Pulmonary embolus diagnosed by endobronchial ultrasound. Respir Med Case Rep 2015; 16: 104-5.

16. Xing XQ, Yang J, Li ZD, Liu YH, Xiao Y, Li YL, et al. Peripheral Pulmonary Emboli Detected by Radial Probe Endobronchial Ultrasound. Chin Med J 2015; 128: 3116-7.

17. Nicoara A, Assaad S, Geirsson A, Rousou A, Jadbabaie F. Unexpected intraoperative diagnosis of pulmonary embolism by transesophageal echocardiography. J Cardiothorac Vasc Anesth 2010; 24: 639-40.

How to cite this article: Zhu R, MaXC. Clinical value of ultrasonography in diagnosis of pulmonary embolism in critically ill patients. J Transl Intern Med 2017; 5: 200-4. 